\title{
多取代呋喃合成最新研究进展
}

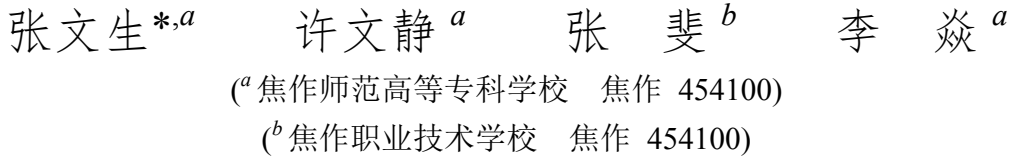

摘要 呋喃是一类重要的五元含氧杂环化合物, 其不仅是许多天然产物、药物和生物活性分子的核心结构, 而且还是 重要的有机合成中间体, 具有广泛的应用价值. 因此, 呋喃及其衍生物的合成一直是有机化学家关注的研究热点之一. 对 2013 年以来多取代呋喃的合成新方法进行了综述，包括二取代、三取代、四取代呋喃和苯并呋喃等.

关键词 呋喃; 合成; 环化反应

\section{Recent Progress in Synthesis of Polysubstituted Furans}

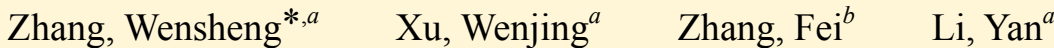 \\ ( ${ }^{a}$ Jiaozuo Teachers College, Jiaozuo 454100) \\ ( ${ }^{b}$ Jiaozuo Vocational and Technological School, Jiaozuo 454100)
}

\begin{abstract}
Furan, an important class of oxygen-containing five-membered heterocyclic compounds, is not only the fundamental structure of many natural products, drugs and biologically active molecules, but also an valuable intermediate in organic synthesis. Synthesis of polysubstituted furans has been one of the focus of organic chemists. In this paper, recent progress in the synthesis of polysubstituted furans since 2013, including disubstituted, trisubstituted, tetrasubstituted furans and benzofurans is reviewed.
\end{abstract}

Keywords furan; synthesis; cyclization

多取代呋喃广泛存在于天然产物和药物中 ${ }^{[1]}$, 同时 也是重要的有机合成反应砌块 ${ }^{[2]}$ 和构建高分子材料的基 础骨架 ${ }^{[3]}$. 因此，发展新型高效的多取代呋喃合成方法 一直是有机合成的热点之一. 对近 5 年来的合成方法新 进展进行了综述.

\section{1 二取代呋喃的合成}

Ramón 课题组 ${ }^{[4]}$ 以芳炔为原料, 首先在 $\mathrm{CuO}$ 负载于 $\mathrm{Fe}_{3} \mathrm{O}_{4}$ 得到的非均相催化剂和 $t$-BuOK 作用下, 通过无溶 剂反应得到 1,3-二炔中间体(Eq. 1). 经回收催化剂再利 用之后，向反应体系中加入 $\mathrm{KOH} / \mathrm{H}_{2} \mathrm{O} / \mathrm{DMSO}$, 在 $80{ }^{\circ} \mathrm{C}$ 下反应 $3 \mathrm{~d}$, 将 1,3-二炔中间体转化为 2,5-二芳基取代的 呋喃. 原料芳炔的苯环上含有 4-甲基或 4-三氟甲基取代 基时，反应收率高于 $90 \%$. 但是苯环上含有甲氧基取代 基，反应收率明显降低(50\%). 脂肪炔在同样的条件下 则不能完成反应.

$$
\begin{aligned}
& \text { One-pot } \\
& \text { (i) } \mathrm{CuO}-\mathrm{Fe}_{3} \mathrm{O}_{4} \text { (2.6 mol\%) } \\
& \mathrm{Ar} \equiv \frac{t \text {-BuOK }(1 \text { equiv. }), 60^{\circ} \mathrm{C}}{\text { (ii) } \mathrm{KOH}(10 \text { equiv. })}
\end{aligned}
$$

Reddy 等 ${ }^{[5]}$ 研究发现, 以炔醛和丙烯酸甲酯为底物 通过 Morita-Baylis-Hillman 反应得到的产物，再和乙酸 之间发生酯化反应得到一种有价值的中间体。该中间体 在 $\mathrm{Pd}\left(\mathrm{PPh}_{3}\right)_{4}$ 催化下，在温和的室温条件下通过 $\mathrm{AcO}$ 基 团的 1,3-迁移异构化、脱酰基和异构化环合三步串联反 应，构建 5-取代呋喃-3-甲酸甲酯类化合物(Scheme 1). 芳炔上的吸电子或供电子基团对反应活性几乎没有影 响，均可以实现高于 $80 \%$ 的收率. 当 $\mathrm{R}$ 为烯基或烷基时, 也可以获得 $64 \% \sim 72 \%$ 的产物.

麻生明等 ${ }^{[6]}$ 通过铜催化的炔丙基叔醇、乙醛酸乙酯

* Corresponding author. E-mail: tongjizws@163.com

Received November 18, 2018; revised December 25, 2018; published online January 18, 2019.

Project supported by the Foundation and Frontier Research Program (the Natural Science Foundation) of Henan Province (No. 162300410270). 河南省基础与前沿技术研究计划(自然科学基金)(No. 162300410270)资助项目 


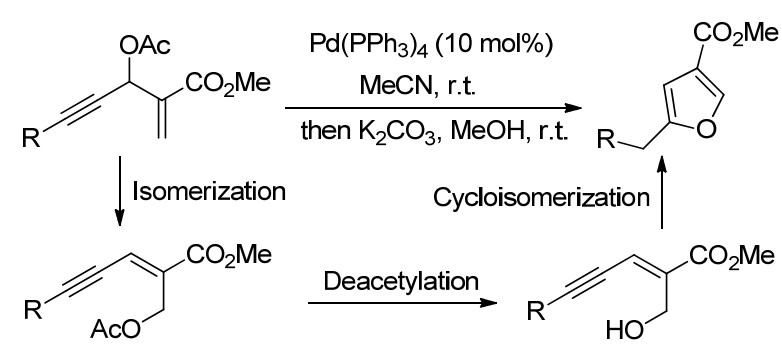

图式 15 -取代呋喃-3-甲酸酯的合成

Scheme 1 Synthesis of 5-substituted furan-3-carboxylates

和仲胺的三组分反应, 在温和条件下得到 2,2,5,5-四取 代的二氢呋喃 (Scheme 2). 以炔丙基仲醇 $\left(\mathrm{R}^{2}=\mathrm{H}\right.$ ) 为底物 时, 该中间体在 $\mathrm{CH}_{3} \mathrm{I}-\mathrm{Cs}_{2} \mathrm{CO}_{3}-\mathrm{MeCN}$ 体系中 $80{ }^{\circ} \mathrm{C}$ 加热, 通过生成季铵盐和消除反应实现芳构化, 可以转化为 2,5-二取代呋喃. 该合成二取代呋喃方法适用于 $R^{1}$ 为烷 基、芳基(含呋喃环或荎环)或烯基的各种二级炔丙醇底 物，产物的分离收率达到 50\% 65\%.

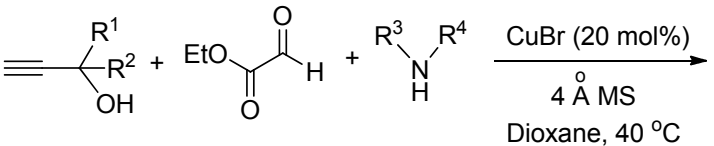

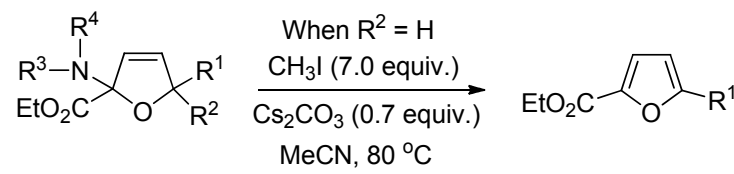

图式 2 以炔、醛和胺为原料 $\mathrm{Cu}(\mathrm{I})$-催化合成 2,5-二取代呋喃 Scheme 2 Copper(I)-catalyzed synthesis of 2,5-disubstituted furans from alkynes, aldehydes and amines

\section{2 三取代呋喃的合成}

张玉红等 ${ }^{[7]}$ 从含 $\alpha$-亚甲基的酮和 $\alpha, \beta$-不饱和酸两种 易得原料出发, 以 $N, N$-二甲基甲酰胺(DMF)为溶剂, 在 $\mathrm{CuCl}-\mathrm{Cu}(\mathrm{OAc})_{2} \cdot \mathrm{H}_{2} \mathrm{O}$ 促进下通过脱羧环化异构化反应, 区域选择性的合成了 2,3,5-三取代呋喃化合物(Scheme 3). 该合成策略对于大多数的底物通用性较好. 但当 $\mathrm{R}^{3}$ 为含有供电子的烷氧基团, 或 $\mathrm{R}^{1}$ 为 $t$ - $\mathrm{Bu} 、 2$-萗基或 2噻吩基等大位阻取代基团时, 产物的收率会明显下降 (低于 $40 \%$ ). Hajra 等 ${ }^{[8]}$ 以同样类型的底物, 在水合乙酸 铜催化下, 通过脱羧环合反应得到 2,3,5-三取代的呋喃. 他们的研究表明, 向反应体系中加入水对该条件下的反 应转化起很关键的作用. 上述合成方法不仅合成原料易 得、催化剂的成本低廉, 而且操作简便, 不需要在特殊 的惰性气体保护下完成.

Hashmi 等 ${ }^{[9]}$ 以 1,4-二炔-3-醇为原料, 通过 $\mathrm{Au}$ 催化 活化得到 $\mathrm{Au}$-卡宾- $\alpha$-酮中间体 $\mathbf{A}$, 再经过 1,2-炔基迁移 后异构化为 1,3-二酮的中间体 $\mathbf{B}$, 最后发生环化后得到

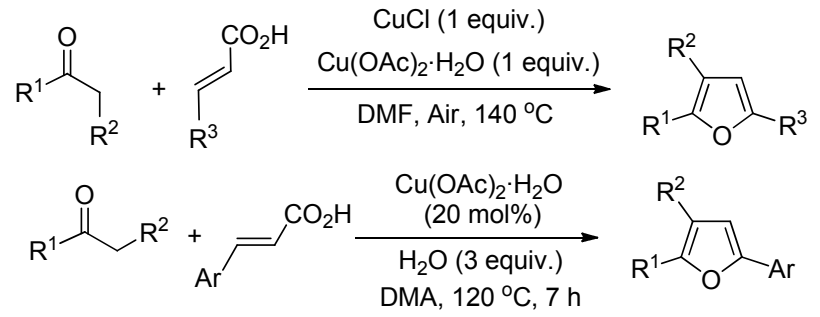

图式 3 以烷基酮和 $a, \beta$-不饱和酸为原料合成 2,3,5-三取代呋 喃

Scheme 3 Synthesis of 2,3,5-trisubstituted furans from alkyl ketones and $a, \beta$-unsaturated carboxylic acids

2,5-二取代呋喃-3-甲醛(Scheme 4)。该合成策略条件温 和(室温), 反应效率高(30 min), 对于 $\mathrm{R}$ 为芳基的各种底 物均有很好的普适性(收率 $80 \% \sim 95 \%$ ); 当 $\mathrm{R}=n$ - $\mathrm{Bu}$ 时， 也可以达到 $63 \%$ 的收率.

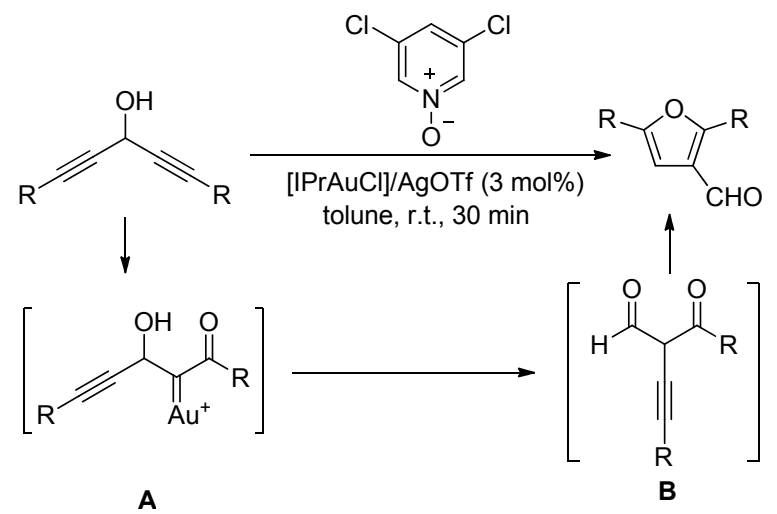

图式 4 多取代-3-甲酰基呋喃的合成

Scheme 4 Synthesis of highly substituted 3-formylfurans

Chuang 等 ${ }^{[10]}$ 研究了 1,3 -二炔二甲酸酯、三取代膦和 芳醛之间的三组分反应, 得到含有活性膦叶立德 $(\mathrm{P}-$ Ylide)取代基的三取代呋喃(Eq. 2). 目标产物中的叶立 德基团可以氧化得到 $\alpha$-酮酸酯官能团, 也可以转化为烯 键. 该反应仅局限于苯环上含吸电子基团的芳醛和三芳 基膦的底物. 亲核性更强的烷基膦以及无吸电子取代基 的苯甲酫和吡啶醛则不能得到预期产物. 对三芳基膦底 物的进一步考察发现, 芳环上含有供电子基团时有利于 活性的提高, 而含有吸电子基团或是杂环代替苯环的底 物则活性相对较差.

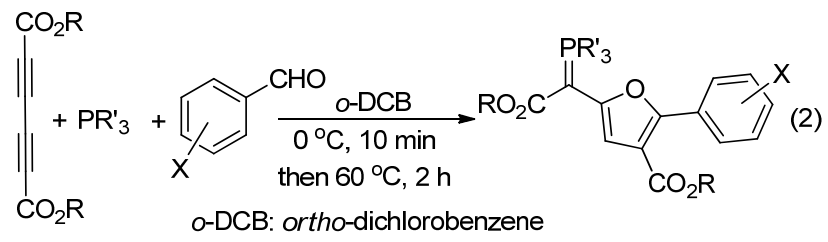

Hajra 等 ${ }^{[11]}$ 报道了一种以 $\alpha$-亚甲基酮和 $\beta$-硝基苯乙 烯为原料，通过 $\mathrm{Cu}(\mathrm{I})$ 催化的分子间环化反应，以中等收 
率区域选择性的合成 2,3,5-三取代呋喃的方法(Eq. 3). 该反应中的酮既可以是芳香(杂环)酮，也可以是甲基酮 或环酮. 原料 $\beta$-硝基苯乙烯中苯环上取代基的电子效应 对反应活性无明显影响.

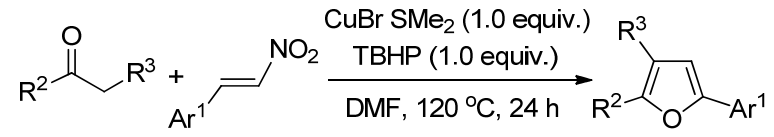

雷爱文等 ${ }^{[12]}$ 选择了 $\mathrm{I}_{2}$ - $\mathrm{CHP}$ (过氧化氢异丙苯)催化 氧化体系调节自由基反应的活性, 完成了 $\beta$-酮酸酯和炔 的氧化偶联和环化异构化的串联反应, 以中等的收率 ( $46 \% \sim 60 \%)$ 得到三取代的呋喃-3-甲酸酯类化合物(Eq. 4). 对底物的通用性考察表明: 脂肪炔和芳炔具有相似 的反应活性, 芳炔苯环上的取代基电子效应对反应收率 也无明显影响, 但是以 1,3 -二酮代替 $\beta$-酮酸酯的反应未 能得到相应的目标产物.

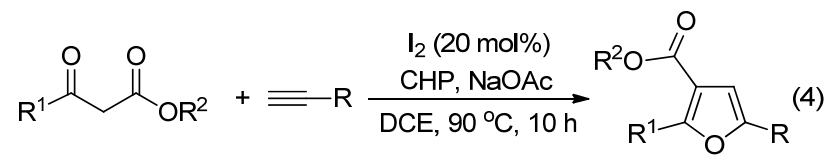

江焕峰课题组 ${ }^{[13]}$ 以含有吸电子基团的共轭炔烯基 酮和二取代磷酸酯为原料, 实现了膦基化的多取代呋喃 构建(Scheme 5). 在 $\mathrm{Cu}(\mathrm{I})$ 催化下, 该两种底物转化为含 $\mathrm{C}\left(\mathrm{sp}^{3}\right)-\mathrm{P}$ 键的 2,3,5-三取代呋喃化合物. 如果在 $\mathrm{Cs}_{2} \mathrm{CO}_{3}$ 促进下, 则构建的是 $2,3,4,5$-四取代的呋喃环.

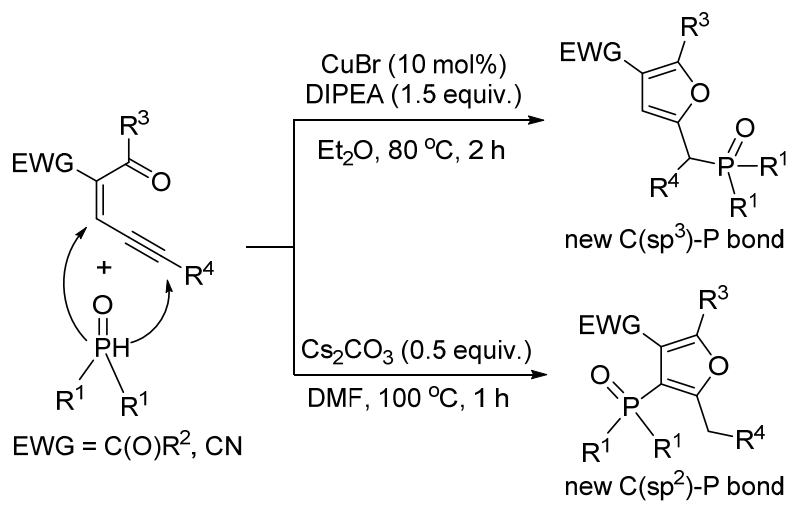

图式 5 膦酰化呋喃的合成

Scheme 5 Synthesis of phosphorylated furans

Hajra 等 ${ }^{[14]}$ 报道了 $\mathrm{Cu}(\mathrm{II})$ 催化下, 在空气介质中通过 芳基酮和芳基乙烯的环化反应构建三取代或四取代呋 喃的方法(Eq. 5). 该方法经历了铜催化的自由基反应历 程, 底物适应性范围较广, 收率高. 以单芳基乙烯为原 料得到的是 2,3,5-三取代呋喃; 若以二芳基乙烯为底物, 则构建的是四取代的呋喃化合物.

Chen 和 $\mathrm{He}$ 等 ${ }^{[15]}$ 以 $\alpha, \beta$-不饱和烯酮和溴代硝基甲烷

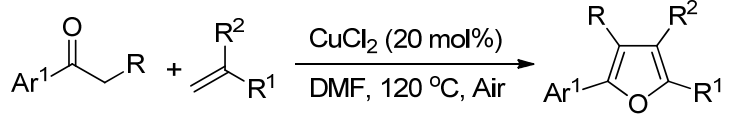

$$
\begin{aligned}
& \mathrm{R}^{1}=\text { Aryl; } \mathrm{R}^{2}=\mathrm{Ph}, \mathrm{H}
\end{aligned}
$$

为原料，合成了一系列的多取代呋喃(Scheme 6). 该反 应在四氢呋喃(THF)中进行, 向反应体系中加入 1 equiv. 的 $\mathrm{K}_{2} \mathrm{CO}_{3}$, 室温搅拌 $12 \mathrm{~h}$, 主要产物是二氢呋喃. 如果 再向反应液中加入 2 equiv. 的 DABCO, 继续室温搅拌 24 $\mathrm{h}$, 进一步脱氢芳构化得到三取代的呋喃.

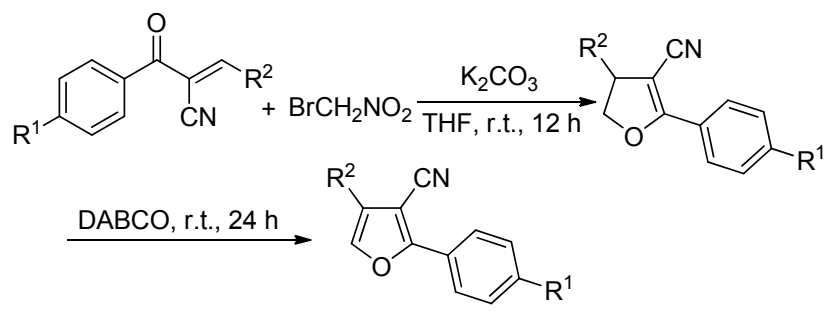

图式 6 以 $\alpha, \beta$-不饱和烯酮和溴代硝基甲烷为原料合成多取代 呋喃

Scheme 6 Synthesis of polysubstituted furansfrom bromonitromethane and oxodienes

我们课题组 ${ }^{[16]}$ 以 1,1 -二溴代芳基乙烯和乙酰乙酸乙 酯为原料, 在 $\mathrm{Ag}_{2} \mathrm{CO}_{3}-\mathrm{Cs}_{2} \mathrm{CO}_{3}-\mathrm{KOAc}$ 体系的促进下, 通 过氧化偶联-环合反应合成了一系列的 2,3,5-三取代呋 喃(Eq. 6). 反应所需的底物 1,1-二溴代芳烯烃可通过芳 醛的 Corey-Fuchs 反应定量的获得. 底物 1,1-二溴代芳 烯在相应的反应条件下首先发生消除生成炔中间体. 该 中间体在 $\mathrm{Ag}$ 促进下和乙酰乙酸乙酯通过 $\mathrm{C}\left(\mathrm{sp}^{3}\right)-\mathrm{H}$ 和 $\mathrm{C}(\mathrm{sp})-\mathrm{H}$ 的氧化偶联发生炔基化、再经历环化过程最终 得到目标产物.

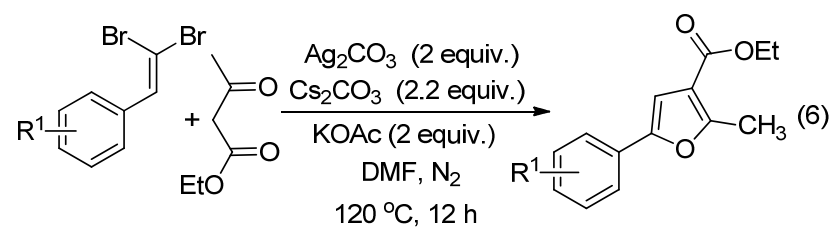

\section{3 四取代呋喃}

Liang 等 ${ }^{[17]}$ 报道了一种环戊烷并多取代呋喃的合成 方法. 该方法以 2-炔基-2-烯-1-酮和 1,1-二芳基乙烯为原 料，通过金催化在室温下反应 $1 \mathrm{~h}$, 得到了该类特殊结 构的多取代呋喃(Eq. 7). 吸电子和供电子取代基对该合 成方法的收率没有明显的影响.

Song 和 You 等 ${ }^{[18]}$ 以端基炔和 1,3-二羰基化合物为 原料，在 $\mathrm{Au}$ 催化下通过 $\mathrm{C}\left(\mathrm{sp}^{3}\right)-\mathrm{H}$ 和 $\mathrm{C}(\mathrm{sp})-\mathrm{H}$ 的氧化 偶联一环化串联反应，得到了一系列的 3-炔基多取代呋 喃类化合物(Eq. 8). 该反应条件温和, 产物的区域选择 


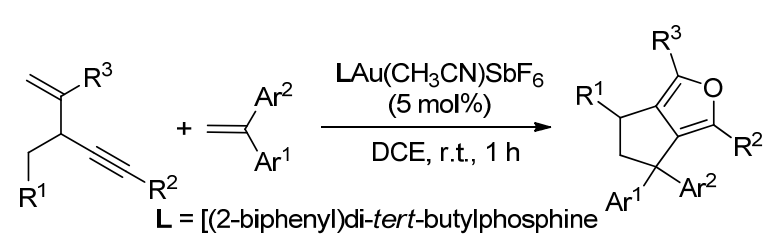

性高, 底物的普适性强, 1,3-二羰基底物的结构对反应 活性没有显著的影响. 芳炔底物的活性则明显高于烷基 炔和含有杂环的炔类.

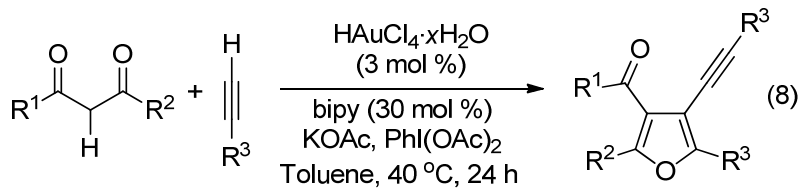

Bach 等 ${ }^{[19]}$ 以甲氧基取代的炔丙醇乙酸酯和三甲硅 基烯醇醚为原料，在 $\mathrm{Bi}(\mathrm{OTf})_{3}$ 催化下合成 2-烯基四取代 呋喃(Scheme 7). 该反应的特点是条件温和, 效率高, 在 $0 \sim 25{ }^{\circ} \mathrm{C}$ 条件下 $5 \mathrm{~min}$ 即可完成转化. 反应所需的底 物炔丙醇乙酸酯可以由端基炔烃为原料, 经过两步反应 制备.
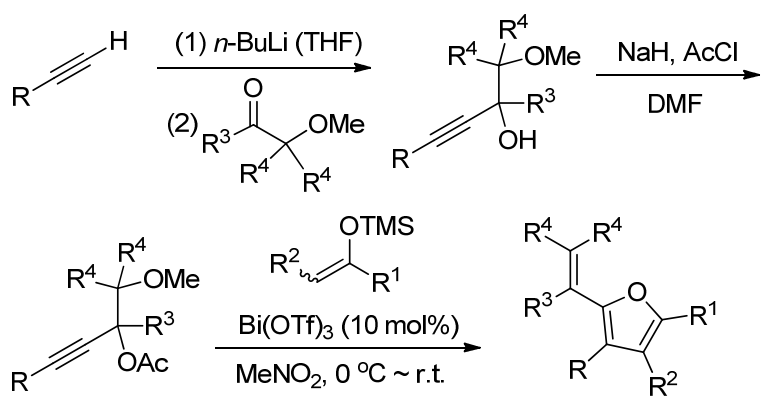

图式 7 多取代-2-烯基呋喃的合成

Scheme 7 Synthesis of substituted 2-alkenylfurans

徐政虎等 ${ }^{[20]}$ 以 $\mathrm{Cu}(\mathrm{OAc})_{2}-\mathrm{Pd}(\mathrm{OAc})_{2}$ 混合 “转移” 催 化, 以 $\mathrm{CH}_{3} \mathrm{CN}-\mathrm{DMSO}$ 为混合溶剂, 在较低的温度和较 短的反应时间将环丙烯衍生物转化为 $2,3,4,5$-四取代呋 喃类化合物(Eq. 9).

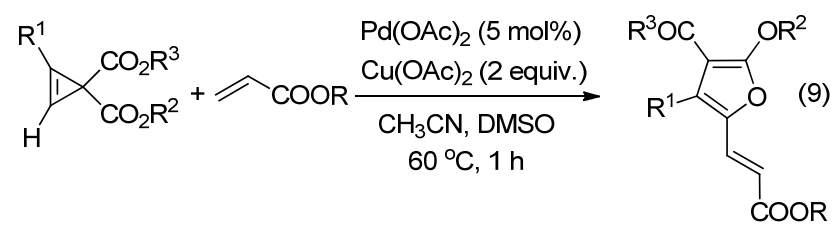

卓克䂞课题组 ${ }^{[21]}$ 以 $\alpha, \beta$-不饱和酮和 1,3-二羰基化合 物为底物, 在 $\mathrm{MnO}_{2}$ 的促进下通过自由基加成和 $\mathrm{C}-\mathrm{O}$ 偶联的闭环反应构建了含 3,4-二酰基的四取代呋喃(Eq. 10). 反应的通用性考察表明: $\alpha, \beta$-不饱和酮底物中的取 代基电子效应对反应转化无显著影响. 1,3-二羰基化合 物底物则既可以是 1,3 -二酮，也可以是 $\beta$-酮酸酯类.

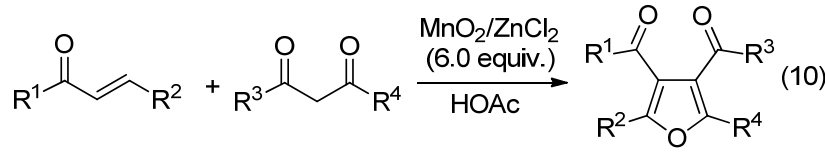

Reddy 等 ${ }^{[22]}$ 报道了一种在有机碱(DABCO 和 DBU) 促进下，通过 $\mathrm{MBH}$ 酯和羰基 $\alpha$-位亚甲基之间的取代反 应和环化异构化构建四取代呋喃环的方法(Eq. 11). 该 合成方法的反应条件温和(室温下完成), 无需金属催化, 且原料易得( $\mathrm{MBH}$ 酯可以通过 Morita-Baylis-Hillman 反 应制备).

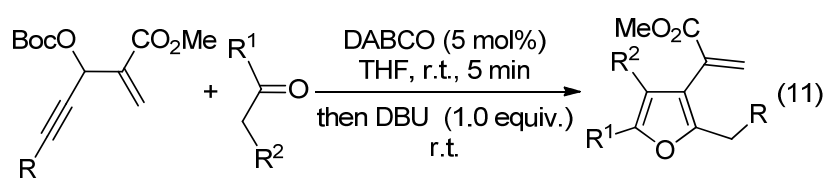

Namboothiri 等 ${ }^{[23]}$ 以 $\alpha$-硝基苯乙酮亲核进攻 $\mathrm{MBH}$ 乙酸酯，在室温条件下以中等收率(45\% 59\%)得到含 硝基的具有区域选择性的四取代呋喃(Eq. 12), 芳环上 的供电子或吸电子取代基对反应收率没有明显的影响, 邻位取代基的位阻效应也不明显. 该方法以 $\mathrm{DABCO}$ 为 碱, 反应过程涉及分子内 Michael 加成和分子内 Mannich 反应等关键步骤.

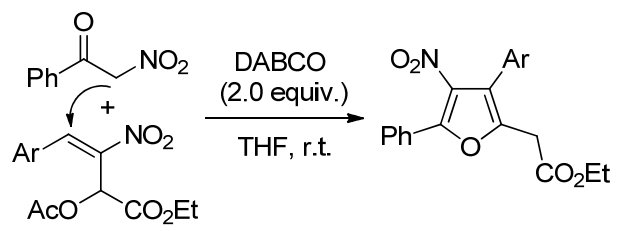

贺峥杰等 ${ }^{[24]}$ 以三丁基膦促进的取代丙炔酸酯、芳香 醛和酰氯四组分环化反应，以中等收率生成四取代的呋 喃(Eq. 13). 脂肪和芳香酰氯都可以参与反应. 大多数的 芳香醛在优化条件下可以得到预期产物，但是脂肪醛在 同样的条件下不能完成反应. 直接以丙炔酸酯为原料 时，则会得到 2,3,5-三取代的呋喃. 该合成方法首先经 过原位生成磷叶立德活性中间体，接着发生分子内 Wittig 反应，再脱去三丁基氧膦得到目标产物. 该反应 使用简单易得的原料，在温和的室温条件下完成，为多 取代呋喃的合成提供了简便的新方法.

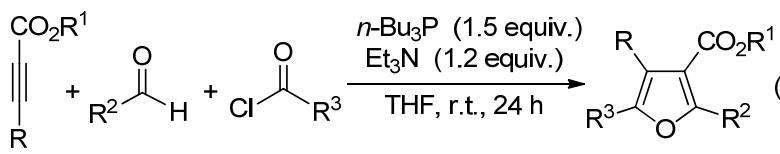

王存德等 ${ }^{[25]}$ 报道了一例 DABCO 促进下, 由 1-腈基 环丙烷-1-甲酸酯和醛之间的开环-环加成反应合成四取 代呋喃的方法，最高收率可达到 $98 \%$ (Eq. 14). 该反应 适用于苯环上含各种供电子和吸电子基团的反应底物, 多数情况下均能取得满意的收率，只有甲氧基的存在会 
导致反应收率稍微下降. 该合成方法无需添加溶剂, 符 合绿色合成的理念. 反应的历程包含了环丙烷开环、分 子间亲核加成、分子内 $O$-亲核加成和芳构化等多个关键 步骤.

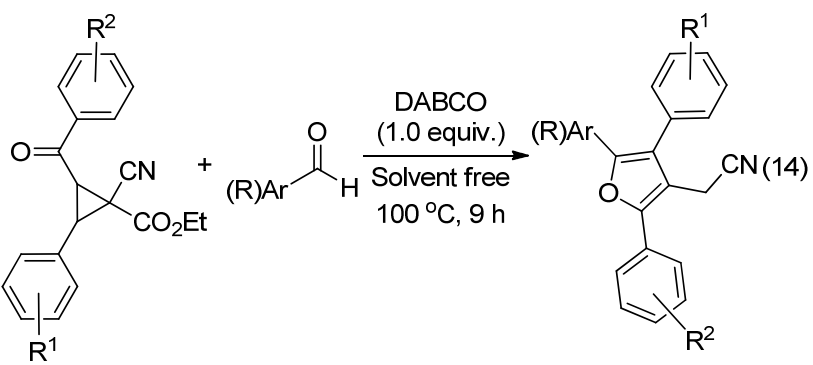

Selander 等 ${ }^{[26]}$ 开发了一种 $\operatorname{In}(\mathrm{III})$ 催化的环戊烷并呋 喃化合物合成方法(Eq. 15). 该反应采用环境友好的 Lewis 酸催化体系, 以 $\alpha$-炔基不饱和酮、醛和仲胺为原 料发生三组分反应, 具有很好的原子经济性. 该方法的 底物普适性也较好, 大多数的反应底物在优化后的条件 下均能获得理想的反应收率和立体选择性.

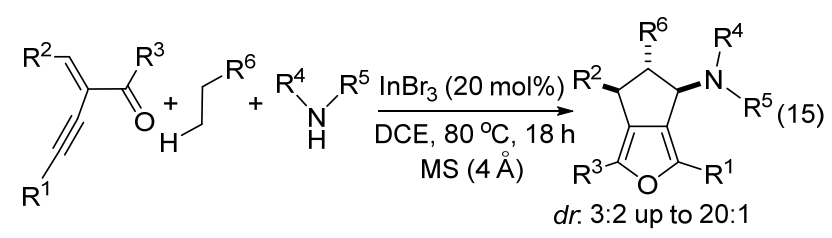

\section{4 苯并呋喃的合成}

Sahoo 等 ${ }^{[27]}$ 以酚和非活化的内炔为原料, 通过 Pd催化环化的加成偶联反应, 构建了多取代的苯并呋喃类 化合物(Eq. 16). 该方法的反应底物普适性宽泛. 酚环上 取代基的电子效应对反应活性无明显的影响, 但是酚差弪 基邻位有取代基时，空间位阻效应则会导致反应收率的 显著降低. 内炔的两个取代基既可以是芳基, 也可以是 烷基. 芳基-烷基混合内炔参与反应时, 产物的区域选 择性很高, 主要得到的是芳基取代基在呋喃环 $\alpha$-位的产 物.

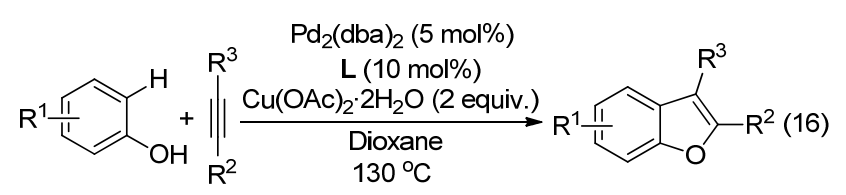

L: bathophenanthroline or 1,10-phenanthroline

Maiti 等 ${ }^{[28]}$ 在相似的 Pd-催化条件下, 利用酚和端基 烯烃之间的偶联环化反应合成 $\alpha$-取代的苯并呋喃, 反应 的收率从中等到较高(Eq. 17). 两种底物苯环上的邻位 取代基的位阻效应不显著. 酚环上吸电子基团的存在不 会降低反应的收率. 因此, 该合成策略提供了一种用其
他方法很难得到的苯环上含吸电子基团苯并呋喃的合 成途径.

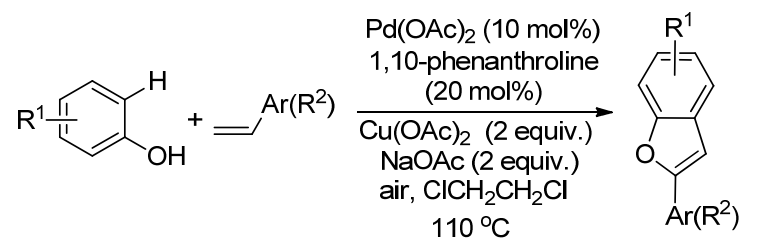

张卫东等 ${ }^{[29]}$ 以邻碘代酚和连有吸电子基团的炔为 原料，通过酚羟基对参键的亲核加成和 Pd 催化的分子 内 Heck 反应两步串联的 “一锅法” 反应，高收率地构 建 2,3-二取代苯并呋喃环(Scheme 8). 芳环和炔两种底 物中的各种取代基团对反应效率均没有明显的影响. 该 合成策略还被成功应用于天然产物 Daphnodorin B 的全 合成.
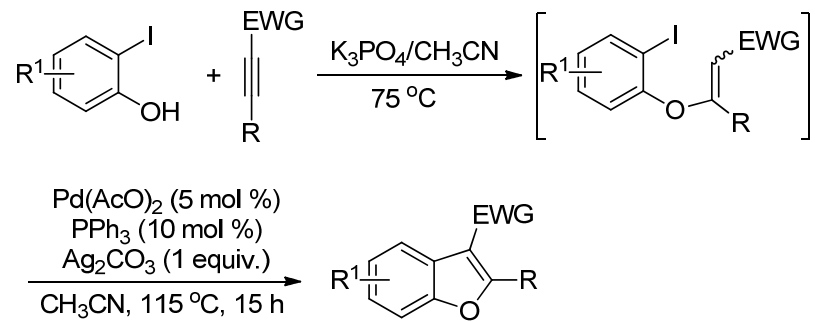

图式 8 3-取代-3-功能化苯并呋喃的合成 Scheme 8 Synthesis of 2-substituted-3-functionalized benzofurans

Larock 等 ${ }^{[30]}$ 以邻碘代酚、端基芳炔和芳基碘为原料, 通过微波促进下和 Pd 催化的三种组分 “一锅” 反应，用 较短的反应时间高收率地转化为 2,3-取代的苯并呋喃化 合物(Eq. 18). 反应效率几乎不受酚环上取代基电子效 应的影响，但是酚羟基邻位有较大体积取代基如烯丙基 等存在时，位阻会导致收率下降. 此外，部分溶解性较 差的底物会影响第一步 Sonogashira 偶联反应的发生, 从而导致两步反应的总收率下降. 底物扩展研究表明: 当芳炔底物中有强吸电子基团如腈基存在时，环合反应 不能发生. 脂肪族端基炔在同样的条件下也无法得到相 应的呋喃产物。

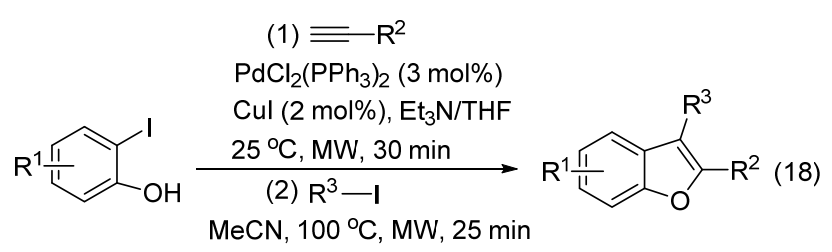

Osyanin 等 ${ }^{[31]}$ 报道了一例合成 2-硝基取代的苯并呋 喃衍生物的新方法. 该反应以三硝基甲烷化钾作为 1,1亲电一亲核两性等价体，和 1,4-两性等价体环合，反应收 率中等(Scheme 9). 该类化合物不仅具有重要的生理活 
性，还是非常有价值的合成中间体. 苯环上的各种供电 子和吸电子基团以及邻位取代基均不会影响反应活性.

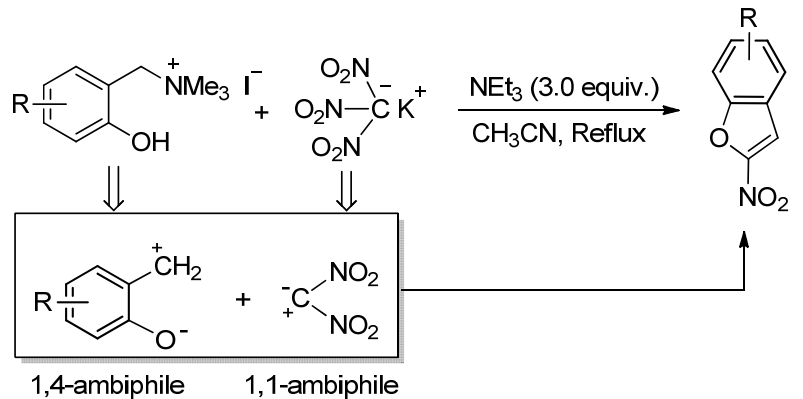

图式 92 -硝基苯并呋喃的合成

Scheme 9 Synthesis of 2-nitroarenofurans

Tsuji 和 Nakamura 等 ${ }^{[32]}$ 以邻炔基酚为原料，通过 BuLi- $\mathrm{ZnCl}_{2}$ 促进的分子内环化反应得到苯并呋喃- $\beta$-氯 化锌中间体. 该中间体质子化后直接得到三取代的呋 喃, 若在低温下接受亲电试剂进攻, 则可以转化为四取 代的呋喃(Scheme 10). 该方法适用于大多数的反应底 物, 转化率最高可接近 $100 \%$.
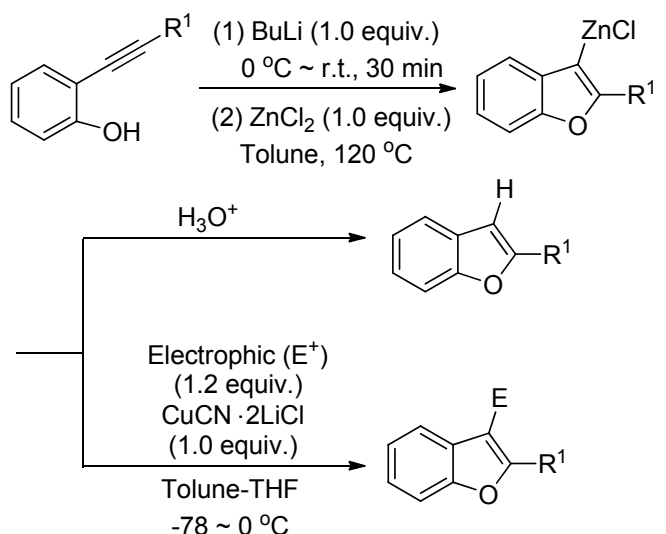

图式 10 以邻炔基酚为原料合成苯并呋喃

Scheme 10 Synthesis of benzofurans from 2-alkynylphenols

\section{5 小结}

近年来, 有机化学家在呋喃衍生物的合成方面做了 大量的研究, 发现了很多新颖的合成方法. 由于呋喃化 合物在天然产物、药物和合成领域有着重要的作用, 开 发新的合成方法, 尤其是从廉价易得的原料出发, 使用 简便的操作和温和的反应条件, 底物通用性和官能团兼 容性宽泛的新方法, 仍将是化学家们持续关注的热点. 通过原子经济性高的多组分反应和串联反应构建含有 各种官能团的多取代呋喃, 是有机化学和绿色化学领域 面临的新挑战.

\section{References}

[1] (a) Hou, X. L.; Yang, Z.; Wong, H. N. C. Prog. Heterocycl. Chem. 2003, $15,167$.

(b) Keay, B. A.; Dibble, P. W. In Comprehensive Heterocyclic Chemistry II, Vol. 2, Eds.: Katritzky, A. R.; Rees, C. W.; Scriven, E. F. V., Elsevier, Oxford, 1997, p. 395.

(c) Suhre, M. H.; Reif, M.; Kirsch, S. F. Org. Lett. 2005, 7, 3925.

(d) Rao, A. U.; Xiao, D.; Huang, X.; Zhou, W.; Fossetta, J.; Lundell, D.; Tian, F.; Trivedi, P.; Aslanian, R.; Palani, A. Bioorg. Med. Chem. Lett. 2012, 22, 1068.

(e) Kumari, N.; Mishra, C. B.; Prakash, A.; Kumar, N.; Mongre, R.; Luthra, P. M. Neurosci. Lett. 2014, 558, 203.

(f) Hasegawa, F.; Niidome, K.; Migihashi, C.; Murata, M.; Negoro, T.; Matsumoto, T.; Kato, K.; Fujii, A. Bioorg. Med. Chem. Lett. 2014, 24, 4266.

[2] (a) Kalaitzakis, D.; Triantafyllakis, M.; Alexopoulou, I.; Sofiadis, M.; Vassilikogiannakis, G. Angew. Chem., Int. Ed. 2014, 53, 13201. (b) Wang, Z.-L.; Li, H.-L.; Ge, L.-S.; An, X.-L.; Zhang, Z.-G.; Luo, X.; Fossey, J. S.; Deng, W.-P. J. Org. Chem. 2014, 79, 1156.

[3] (a) Zeng, C.; Seino, H.; Ren, J.; Hatanaka, K.; Yoshie, N. Macromolecules 2013, 46, 1794.

(b) Zeng, C.; Seino, H.; Ren, J.; Hatanaka, K.; Yoshie, N. Polymer 2013, 54, 5351.

(c) Gidron, O.; Shimon, L. J. W.; Leitus, G.; Bendikov, M. Org. Lett. 2012, 14, 502.

(d) Gidron, O.; Dadvand, A.; Sheynin, Y.; Bendikov, M.; Perepichka, D. F. Chem. Commun. 2011, 47, 1976.

(e) Bunz, U. H. F. Angew. Chem., Int. Ed. 2010, 49, 5037.

(f) Gidron, O.; Diskin-Posner, Y.; Bendikov, M. J. Am. Chem. Soc. 2010, 132, 2148

[4] Pérez, J. M.; Cano, R.; Yus, M.; Ramón, D. J. Synthesis 2013, 45, 1373.

[5] Reddy, C. R.; Krishna, G.; Reddy, M. D. Org. Biomol. Chem. 2014, 12, 1664.

[6] Fan, W.; Ma, S. M. Eur. J. Org. Chem. 2015, 3531.

[7] Yang, Y. Z.; Yao, J. Z.; Zhang, Y. H. Org. Lett. 2013, 15, 3206.

[8] Ghosh, M.; Mishra, S.; Monir, K.; Hajra, A. Org. Biomol. Chem. 2015, 13, 309 .

[9] Wang, T.; Shi, S.; Hansmann, M. M.; Rettenmeier, E.; Rudolph, M.; Hashmi, A. S. K. Angew. Chem., Int. Ed. 2014, 53, 3715.

[10] Deng, J. C.; Chuang, S. C. Org. Lett. 2014, 16, 5792.

[11] Ghosh, M.; Mishra, S.; Hajra, A. J. Org. Chem. 2015, 80, 5364.

[12] Tang, S.; Liu, K.; Long, Y.; Qi, X. T.; Lan, Y.; Lei, A. W. Chem Commun. 2015, 8769.

[13] Yu, Y.; Yi, S. G.; Zhu, C. L.; Hu, W. G.; Gao, B. J.; Chen, Y.; Wu, W. Q.; Jiang, H. F. Org. Lett. 2016, 18, 400.

[14] Dey, A.; Ali, M. A.; Jana, S.; Hajra, A. J. Org. Chem. 2017, 82, 4812.

[15] Chen, R. S.; Fan, X.; Xu, Z. Z.; He, Z. J. Tetrahedron Lett. 2017, 58,3722 .

[16] Zhang, W. S.; Xu, W. J. Chem. Heterocycl. Compd. 2017, 53, 615.

[17] He, T.; Gao, P.; Qiu, Y. F.; Yan, X. B.; Liu, X. Y.; Liang, Y. M. RSC $A d v$. 2013, 13, 19913.

[18] Ma, Y. H.; Zhang, S.; Yang, S. P.; Song, F. J.; You, J. S. Angew. Chem., Int. Ed. 2014, 53, 7870.

[19] Nitsch, D.; Bach, T. J. Org. Chem. 2014, 79, 6372.

[20] Song, C. L.; Wang, J. W.; Xu, Z. H. Org. Biomol. Chem. 2014, 12, 5802.

[21] Yue, Y. Y.; Zhang, Y. L.; Song, W. W.; Zhang, X.; Liu, J. M.; Zhuo, K. L. Adv. Synth. Catal. 2014, 356, 2459.

[22] Reddy, C. R.; Mohammed, S. Z.; Kumaraswamy, P. Org. Biomol. Chem. 2015, 13, 8310.

[23] Mane, V.; Kumar, T.; Pradhan, S.; Katiyar, S.; Namboothiri, I. N. N. RSC Adv. 2015, 5, 69990.

[24] Zou, W.; He, Z. R.; He, Z. J. Chin. J. Org. Chem. 2015, 35, 1739 (in Chinese).

(邹雯，贺峥嵘，贺峥杰，有机化学, 2015, 35, 1739.) 
[25] Liu, J. M.; Ye, W. J.; Qing, X. S.; Wang, C. D. J. Org. Chem. 2016, 81,7970 .

[26] Pathipati, S. R.; Werf, A.; Eriksson, L.; Selander, N. Angew. Chem., Int. Ed. 2016, 55, 11863.

[27] Kuram,M. R.; Bhanuchandra, M.; Sahoo, A. K. Angew. Chem., Int. Ed. 2013, 52, 4607.

[28] Sharma, U.; Naveen, T.; Maji, A.; Manna, S.; Maiti, D. Angew. Chem., Int. Ed. 2013, 52, 12669.
[29] Yuan, H.; Bi, K. J.; Li, B.; Yue, R. C.; Ye, J.; Shen, Y. H.; Shan, L.; Jin, H. Z.; Sun, Q. Y.; Zhang, W. D. Org. Lett. 2013, 15, 4742.

[30] Markina, N. A.; Chen, Y.; Larock, R. C. Tetrahedron 2013, 69, 2701.

[31] Osyanin, V. A.; Osipov, D. V.; Demidov, M. R.; Klimochkin, Y. R. N. J. Org. Chem. 2014, 79, 1192.

[32] Tsuji, H.; Ilies, L.; Nakamura, E. Synlett 2014, 25, 2099.

(Lu, Y.) 